\section{The European Association of Cardiovascular Imaging/Heart Failure Association Cardiac Oncology Toxicity Registry: long-term benefits for breast cancer treatment}

\author{
“A careful cardiac and oncological risk factor evaluation should allow for \\ more individualized treatment planning; it is important to better predict \\ patients at risk for cardiotoxicity."
}

\section{Guy Jerusalem ${ }^{* 1}$, Marie Moonen², Pierre Freres' \& Patrizio Lancellotti ${ }^{2}$}

The prognosis of breast cancer has improved over time due to earlier diagnoses as a result of screening programs and patient awareness and the increased use of more effective systemic therapies. Oncologists are more and more concerned with the long-term side effects of some anticancer treatments, which should be carefully evaluated. Aromatase inhibitors are associated with an increased risk of osteoporosis, tamoxifen is associated with uterine cancer and chemotherapy is associated with myelodysplasia and secondary leukemia. In addition, long-term cardiac toxicity is an important issue in breast cancer survivors [1]. Since many patients with early-stage breast cancer can be cured by surgery and radiotherapy alone or with the addition of systemic therapy without cardiotoxicity, it is important that the benefits associated with potential cardiotoxic regimens outweigh the risks of short-term and long-term toxicity. Left ventricular (LV) dysfunction has become a common cause of death in breast cancer survivors. Oncological and cardiac risk factors must be carefully evaluated when deciding on adjuvant treatment regimens for breast cancer.

\section{Cancer survivors \& long-term cardiotoxicity}

It has long been known that chemotherapy and radiotherapy - either alone or in combination - exposes a patient to an increased risk of cardiac morbidity and mortality [2]. Anti-HER2 therapies such as trastuzumab are also associated with cardiotoxicity [3]. Anthracycline-related cardiotoxicity (cardiotoxicity type 1) is generally considered to be irreversible, cumulative and dosedependent; type 2 cardiotoxicity related to trastuzumab is more reversible.

Long-term follow-up of 51,246 patients suffering from T1a,bN0M0 breast cancer registered in the SEER Program from 1988 to 2001 revealed that more patients died from heart disease $(n=1727)$ than from breast cancer $(n=1340)$ [4]. This study also revealed that the risk of dying from other causes was five-times higher than the risk of dying from breast cancer 10 years after diagnosis. It is important to keep in mind that in the general population a woman

\section{KEYWORDS}

- anthracyclines $\bullet$ breast cancer

- cardiac toxicity • cardio-oncology

- monitoring • trastuzumab
'Medical Oncology, CHU Sart Tilman Liege \& Liege University, Domaine Universitaire du Sart Tilman, B35, 4000 Liege Belgium

¿University of Liège Hospital, GIGA Cardiovascular Sciences, Cardio-Oncology Clinic Unit, CHU Sart Tilman Liege \& Liege University, Domaine Universitaire du Sart Tilman, B35, 4000 Liege, Belgium

*Author for correspondence: g.jerusalem@chu.ulg.ac.be 


\section{“The long-term cardiac morbidity and mortality benefits of this multidisciplinary cardio-oncology approach should be carefully evaluated."}

was 3.9-times more likely to die from cardiac heart disease than from breast cancer in 2009 [5]. However, age is an important risk factor: in the youngest group of patients aged 25-44 years, women were about three-times more likely to die from breast cancer than cardiac heart disease. Only in postmenopausal women was the risk of dying from cardiac heart disease higher than the risk of dying from breast cancer.

\section{Cardio-oncology}

Cardio-oncology is a new subspeciality in the field of cardiology in which cardiologists join a multidisciplinary team devoted to treating breast cancer. Before starting treatment, it is important to identify patients at increased risk for cardiac toxicity; age is not the only risk factor [6]. Data suggest an association between cardiovascular risk factors such as a history of hypertension, diabetes or known coronary artery disease and cardiotoxicity of anticancer therapy $[7,8]$. Known risk factors for trastuzumab-associated cardiac toxicity also include a lower screening LV ejection fraction (LVEF) and a lower post-anthracycline LVEF [9].

Alternative less cardiotoxic regimens should be considered according to oncological risk if the patients present a high cardiac risk, and more frequent cardiac monitoring is necessary in these patients if cardiotoxic drugs cannot be avoided.

\section{Many unanswered questions}

Nevertheless, many questions still remain. The European Association of Cardiovascular Imaging and Heart Failure Association Cardiac Oncology Toxicity registry is a prospective, multicenter, observational research program of the European Society of Cardiology in breast cancer patients [10] intended for comprehensive data collection and evaluation. More than 13 countries are involved in the pilot study, and all European countries will be invited to participate to the long-term registry. The registry will include information concerning the diagnosis and management of anticancer drug related cardiotoxicity in breast cancer patients and, in particular, the tests performed routinely at baseline, during treatment and during follow-up. Clear guidelines for monitoring and therapy interruption exist in particular for adjuvant therapy with trastuzumab. Treatment by $\beta$-blockers and angiotensin-converting enzyme inhibitors or angiotensin receptor blockers is necessary in the case of asymptomatic decrease of LVEF. It has been reported that trastuzumab can be successfully reintroduced in many patients after interruption without observing a new event of cardiotoxicity [11], which should also be confirmed in a large unselected patient population.

\section{Who is eligible for this cardiac registry?}

All breast cancer patients who will receive or are receiving potential cardiotoxic anticancer therapies and who underwent a cardiologic imaging technique are eligible. Taratini et al. [12] observed a higher prevalence of hypertension, diabetes, renal dysfunction and dyslipidemia in patients older than 60 years compared with younger patients in a multicenter Italian trial evaluating trastuzumab-associated cardiotoxicity. Poor cardiac outcome in an older patient population has also been reported by ChavezMacGregor et al. [13] in an even larger population-based study using the SEER-Medicare and the Texas Cancer Registry-Medicare databases. These authors observed a very high rate of congestive heart failure in women at least 66 years old who suffered from early-stage breast cancer and who were treated by chemotherapy.

\section{What are the collected variables in the registry?}

The following information will be collected from all patients: demographic characteristics, co-morbidities and risk factors for cardiovascular diseases, cancer history, current treatment and expected anticancer therapy including breast surgery, radiotherapy, chemotherapy, targeted therapy and endocrine therapy, clinical signs and symptoms of cardiotoxicity and results of monitoring tests including biomarkers such as troponin and BNP and cardiac imaging techniques including echocardiography, stress tests, MUGA and MRI. The use of cardioprotective agents and the pharmacological treatments of cardiotoxicity will also be reported.

\section{Is this registry just another registry?}

The current project is just the first step of a much more ambitious project. Additional knowledge should be made available by the registry concerning predisposing cardiac risk factors, the rate of subclinical LV dysfunction in the real world for the most frequently administered cardiotoxic anticancer agents, and transition to overt heart failure. In addition, the registry will further evaluate the clinical impact and outcome of anticancer drug related cardiotoxicity. This 
project focuses on breast cancer, but the treatment of other oncological diseases also involves cardiotoxic drugs. In the future, these cardiooncology clinics should be available for all cancer patients who are candidates for receiving cardiotoxic drugs.

\section{Preparing for the future}

A careful cardiac and oncological risk factor evaluation should allow for more individualized treatment planning; it is important to better predict patients at risk for cardiotoxicity. The aim of this registry is to better understand the risk of cardiac toxicity based on the patient's characteristics prior to treatment and to use this information for better treatment planning in the future. Potential reversibility of cardiac toxicity also needs to be taken into account in particular if early detection is associated with the best chances for complete recovery after appropriate management.

Routinely measuring LVEF before, during and after adjuvant therapy, is standard of care for trastuzumab-based anticancer therapy but many other cardiotoxic drugs are used in the field of breast cancer. The aim of the registry is to also obtain better information concerning the routine cardiac monitoring when other agents are used in order to allow to define clear recommendation also for these other anticancer drugs.

We also need information concerning the use of biomarkers in addition to the use of imaging procedures. The potential benefits of biomarkers include earlier detection of cardiotoxicity, the assessment of physiopathology and the prediction of outcomes. Currently, troponin and BNP are the most frequently used biomarkers, but other biomarkers will also likely be used in the near future. The sensitivity and specificity of biomarkers is a critical issue. If a standard cut-off value of BNP $(>100 \mathrm{pg} / \mathrm{ml})$ is used, many patients presenting later drug-related cardiotoxicity will not be identified. On the other hand, decreasing the cut-off value is associated with many false-positive results [14].

One open question is the role of angiotensinconverting enzyme inhibitors in the prevention of trastuzumab-related cardiotoxicity [15]. $\beta$-blockers are other candidates for preventive treatments [16], and preventive treatments should also be evaluated. The role of preventive or early pharmacological interventions with $\beta$-blockers and/or angiotensin-converting enzyme inhibitors has to be further clarified. Randomized trials are already ongoing in this field [17].

Today, oncologists work together mainly to detect and manage cardiotoxicity, but cardiologists are rarely involved in the initial treatment planning. In the future, the development of cardio-oncology units should make it possible to include cardiologists in treatment planning. Strategies have been developed for decreasing cardiac events by adapting the treatments. For the systemic therapy of HER2-positive breast cancer, we have the choice of using less-cardiotoxic approaches (e.g., by not using anthracyclines or by using sequential regimens of chemotherapy followed by trastuzumab). A careful cardiological and oncological risk evaluation within a multidisciplinary team before starting any systemic therapy should become standard care in order to optimize the chances of recovery without long-term cardiac side effects. This multidisciplinary approach may also help to define common strategies to address new forms of prevention and treatment of anticancer drug associated cardiotoxicity. The long-term cardiac morbidity and mortality benefits of this multidisciplinary cardio-oncology approach should be carefully evaluated.

\section{Financial \& competing interests disclosure}

The authors have no relevant affliations or financial involvement with any organization or entity with a financial interest in or financial conflict with the subject matter or materials discussed in the manuscript. This includes employment, consultancies, honoraria, stock ownership or options, expert testimony, grants or patents received or pending, or royalties.

No writing assistance was utilized in the production of this manuscript.

\section{Open access}

This work is licensed under the Creative Commons Attribution-NonCommercial 4.0 Unported License. To view a copy of this license, visit http://creativecommons.org/ licenses/by-nc-nd/4.0/

\section{References}

1 Bowles EJ, Wellman R, Feigelson HS et al. Risk of heart failure in breast cancer patients after anthracycline and trastuzumab treatment: a retrospective cohort study. J. Natl Cancer Inst. 104(17), 1293-1305 (2012).

2 heart failure in patients treated with doxorubicin: a retrospective analysis of three trials. Cancer 97(11), 2869-2879 (2003).

3 Bird BR, Swain SM. Cardiac toxicity in breast cancer survivors: review of potential cardiac 
problems. Clin. Cancer Res. 14(1), 14-24 (2008).

4 Hanrahan EO, Gonzalez-Angulo AM, Giordano SH et al. Overall survival and cause-specific mortality of patients with stage T1a,bN0M0 breast carcinoma. J. Clin. Oncol. 25(31), 4952-4960 (2007).

5 Canto JG, Kiefe CI. Age-specific analysis of breast cancer versus heart disease mortality in women. Am. J. Cardiol. 113(2), 410-411 (2014).

6 Accordino MK, Neugut AI, Hershman DL. Cardiac effects of anticancer therapy in the elderly. J. Clin. Oncol. 32(24), 2654-2661 (2014).

7 Grenier MA, Lipshultz SE. Epidemiology of anthracycline cardiotoxicity in children and adults. Semin. Oncol. 25(4 Suppl. 10), 72-85 (1998).

8 Yeh ET. Cardiotoxicity induced by chemotherapy and antibody therapy. Annu. Rev. Med. 57, 485-498 (2006).

9 Tan-Chiu E, Yothers G, Romond E et al. Assessment of cardiac dysfunction in a randomized trial comparing doxorubicin and cyclophosphamide followed by paclitaxel, with or without trastuzumab as adjuvant therapy in node-positive, human epidermal growth factor receptor 2-overexpressing breast cancer: NSABP B-31. J. Clin. Oncol. 23(31), 7811-7819 (2005).

10 Lancellotti P, Anker SD, Donal E et al. EACVI/HFA Cardiac Oncology Toxicity Registry in breast cancer patients: rationale, study design, and methodology (EACVI/ HFA COT Registry)-EURObservational Research Program of the European Society of Cardiology. Eur. Heart J. Cardiovascular Imaging 16(5), 466-470 (2015).

11 Ewer MS, Vooletich MT, Durand JB et al. Reversibility of trastuzumab-related cardiotoxicity: new insights based on clinical course and response treatment. J. Clin. Oncol. 23(31), 7820-7826 (2005).

12 Tarantini L, Gori S, Faggiano P et al. Adjuvant trastuzumab cardiotoxicity in patients over 60 years of age with early breast cancer: a multicenter cohort analysis. Ann. Oncol. 23(12), 3058-3063 (2012).

13 Chavez-MacGregor M, Zhang N et al. Trastuzumab- related cardiotoxicity among older patients with breast cancer. J. Clin. Oncol. 31(33), 4222-4228 (2013).
14 Skovgaard D, Hasbak P, Kjaer A. BNP predicts chemotherapy-related cardiotoxicity and death: comparison with gated equilibrium radionuclide ventriculography. PLoS ONE 9(5), e96736 (2014).

15 Hahn VS, Lenihan DJ, Ky B. Cancer therapy-induced cardiotoxicity: basic mechanisms and potential cardioprotective therapies. J. Am. Heart Assoc. 3(2), e000665 (2014).

16 Kalay N, Basar E, Ozdogru I et al. Protective effects of carvedilol against anthracyclineinduced cardiomyopathy. J. Am. Coll. Cardiol. 48(11), 2258-2262 (2006).

17 Pituskin E, Haykowsky M, Mackey JR et al. Rationale and design of the Multidisciplinary Approach to Novel Therapies in Cardiology Oncology Research Trial (MANTICORE 101 - Breast): a randomized, placebo-controlled trial to determine if conventional heart failure pharmacotherapy can prevent trastuzumabmediated left ventricular remodeling among patients with HER $2^{+}$early breast cancer using cardiac MRI. BMC Cancer 11, 318 (2011). 